\title{
Preparation and performance optimization of TPBISi green- light organic luminescent material devices
}

\author{
Huajing Zheng ${ }^{a}$, Quan Jiang, Yadong Jiang and Ruan Zheng \\ State Key Laboratory of Electronic Thin Films \&Integrated Devices, School of Optoelectronic, University of \\ Electronic Science and Technology of China, Chengdu 610054, China
}

\begin{abstract}
The Study analyzed and tested the absorption spectrum, photoluminescence spectrum, and device's electroluminescence spectrum of a new silole material. The device with Silol as an emitting layer, emitted green-light whose structure is ITO/NPB/2,2,3,3-tetraphenyl4,4-bisthienylsilole(TPBTSi)/Alq3/Mg: A by improvement of preparation technology and optimization of thin film. It reaches the maximum luminescence of $11290.2 \mathrm{~cd} / \mathrm{m}^{2}$, the maximum luminous efficiency of $0.84 \mathrm{~lm} / \mathrm{W}$, luminescence spectrum of $516 \mathrm{~nm}$, chromaticity diagram CIE coordinate of $(0.275,0.4568)$ when voltage is $15 \mathrm{~V}$. All of the above is the green characteristic spectrum of TPBTSi.
\end{abstract}

\section{Introduction}

In recent years, OLED has attracted people's wide attention [1-5]. The materials of OLED are divided into two parts such as conjugate polymer materials and small molecule materials. The small molecule materials have definite chemical structure and better color purity, but are easy to crystallize which has become the main factor to shorten the life expectancy of the device. The polymer molecule materials can be processed easily but are hard to purify. In order to solve this problem, scientists have developed star shaped helical molecules containing heteroatom [6]. Compared to the frequently-used heterocyclic compounds containing $\mathrm{N}$, the heterocyclic compound containing $\mathrm{Si}$ (Silole) has lower LUMO energy level, attributed to Silol's special electronic structure. The energy level of LUMO is mixed with $\sigma^{*}$ orbit on Silicon atom and $\pi^{*}$ orbit on part of butadiene[7]. In 1996, 2,5-diarylsiloles 20a-f was first applied to electronic transport materials, and thereafter came the silole derivatives (21a and 21b, 22a-c) in succession. The $20 \mathrm{~d}$ film precipitated under vacuum performs fast and non-dispersion electron transport ( $\mu_{e} \approx 2 \times 10-4 /$ in comparison to the environment of $6.4 \times 105 \mathrm{~V} / \mathrm{cm}$ ). Even in the presence of oxygen, the $20 \mathrm{~d}$ film produces very little electron trap. The electron transport speed rate of $20 \mathrm{~d}$ film is 100 times more than that of Alq3, due to the fact that the Alq3 produces electron trap and leads to dispersion electron transport. Compered to Alq3, to a certain extent, the $20 \mathrm{~d}$ has a larger LUMO value $(3.3 \mathrm{~V})$, that results in better characteristics on electron transport. However, in electrochemical research, most Silole derivatives are irreversibly oxidized and reduced. Inserting $20 \mathrm{~d}$ between $\mathrm{LiF} / \mathrm{Al}$ cathode and Alq3 light emitting layer or 20d can improve device EL efficiency (from 1.9 to $2.6 \mathrm{~lm} / \mathrm{W}$ ). Due to the fact that neither the $\operatorname{Tm}\left(175^{\circ} \mathrm{C}\right)$ or $\mathrm{Tg}$ of $20 \mathrm{~d}$ are high, the surface morphology is damaged

\footnotetext{
${ }^{a}$ Corresponding author : zhj12@163.com

This work was supported by Fund project: National Natural Science Fund (20674049).
} 
by crystalizing while the device is working. The method to improve the $\mathrm{Tg}$ (the highest to $81^{\circ} \mathrm{C}$ ) is to increase the number of substituent aromatic, such as compounds $20 \mathrm{c}-\mathrm{e}$. However, since the Tg of $20 \mathrm{c}-$ e is lower than Alq3, the device prepared by $20 \mathrm{~d}$ for ETM tested under air environment has a better stability. This is probably due to the fact that $20 \mathrm{~d}$ has a better stability under air. Apart from being used as an ETM, Silole (20a and 20f) can also be used as a luminescent material [8-10].

Luminescence mechanism is discussed in this study through four types of Silole complexes 2,2,3,3-tetraphenyl-4,4-bisthienylsilole(TPBTSi) being used as emitting layer, by choosing hole transport layer material NPB and electronic transport layer material $\mathrm{Alq}_{3}$ used as hole and electronic transports layers, preparing high brightness electroluminescence device with simple structure by vacuum thermal evaporation method, charactering device's photoelectric properties and by the analysis of energy level structure.

\section{Test}

\subsection{TPBTSi film surface analysis}

Figure1, 2 show the absorption and emission spectra tested on quartz glass. Figure 3 outlines the surface morphology. From Figure1, the maximum absorption of TPBTSi solid film is $246 \mathrm{~nm}$, the PL peak is $498 \mathrm{~nm}$, which are the characteristics of green light. .

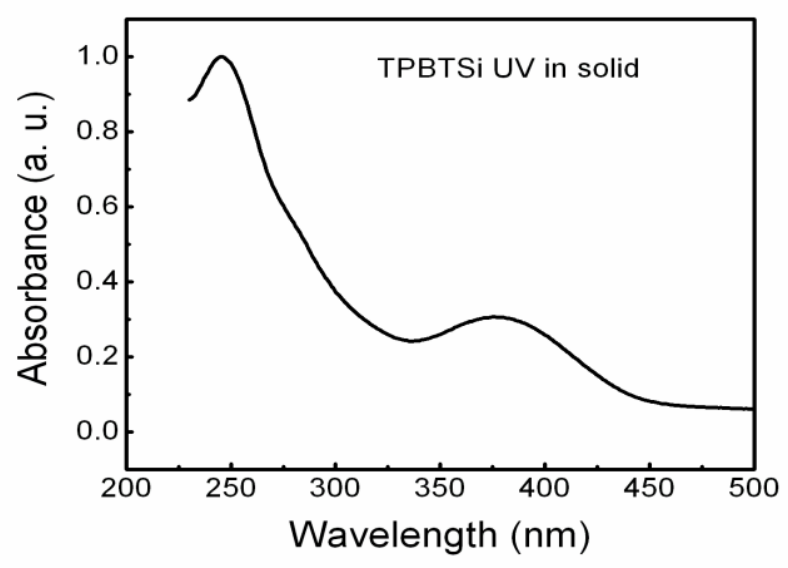

Figure 1. TPBTSi film absorption spectrogram. 


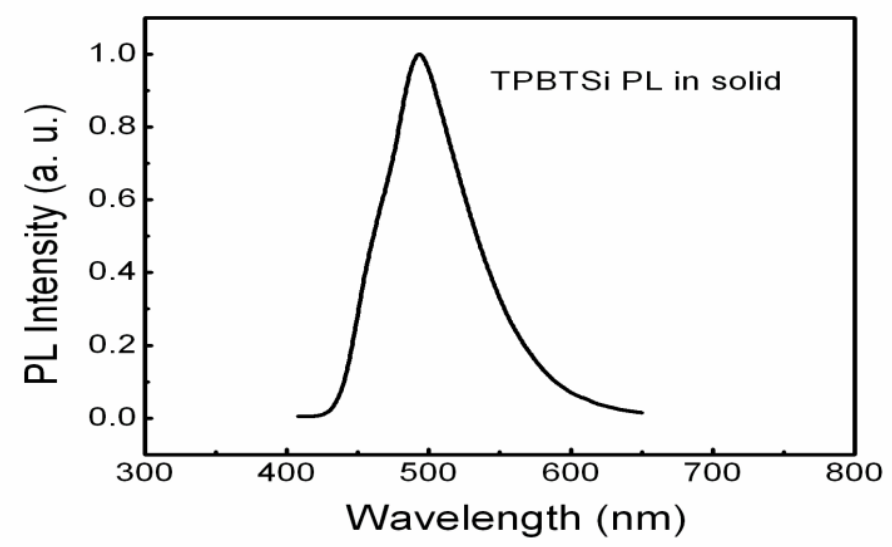

Figure 2. TPBTSi film luminescence spectrogram.

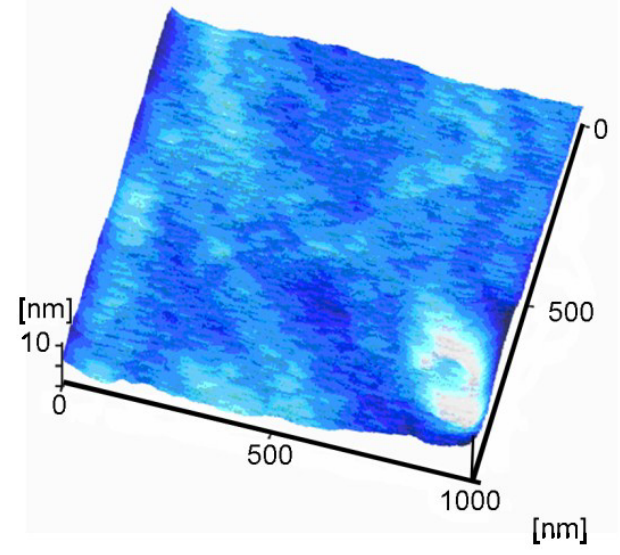

$20 \mathrm{~nm}$ TPBTSi film

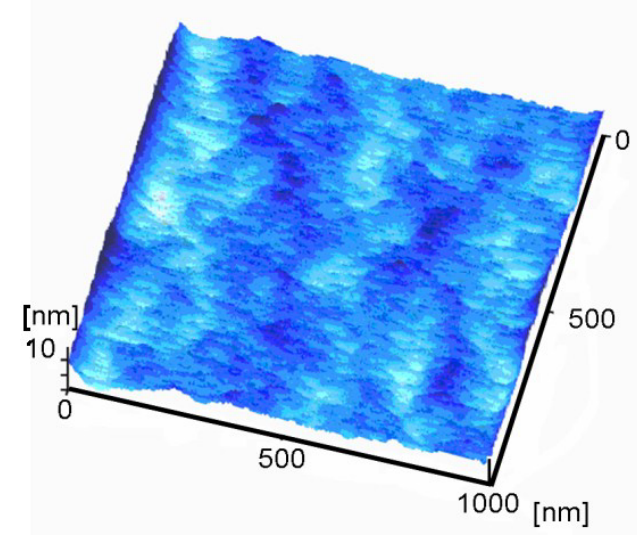

$30 \mathrm{~nm}$ TPBTSi film

Figure 3. TPBTSi film surface morphology.

Table 1 outlines the surface morphology parameters of two substrates and the data shows clearly that the $R_{P-V}$ of $20 \mathrm{~nm}$ film is obviously higher than that of $30 \mathrm{~nm}$. Figure 3 shows that the film with such thickness has pinhole. Both the average roughness $\left(R_{a}\right)$ and the range from peak to trough $\left(R_{\mathrm{P}-\mathrm{V}}\right.$ decrease significantly, meanwhile surface particle radium decreases in at a different degree. The decreas of average roughness and surface particle radium are obviously beneficial to increasing the contact area for the film with other organic layers, which is beneficial to them in terms of attaching closely. In order to use TPBTSi film worked as emitting layer, the electronic and the hole must be controlled in this layer to compound luminescence. In order to avoid the carrier loss caused by the increase of turn-on voltage and the film disadvantage, the thickness of emitting layer TPBTSi should be $30 \mathrm{~nm}$.

Table 1. TPBTSi film surface morphology parameters.

\begin{tabular}{lccc}
\hline \multirow{2}{*}{ substrate } & \multicolumn{3}{c}{ Surface morphology parameters } \\
\cline { 2 - 4 } & $R_{\mathrm{a}}(\mathrm{nm})$ & $R_{\mathrm{P}-\mathrm{V}}(\mathrm{nm})$ & $R_{\mathrm{rms}}(\mathrm{nm})$ \\
\hline $1^{\#} 20 \mathrm{~nm}$ & 0.74 & 5.67 & 1.01 \\
$2^{\#} 30 \mathrm{~nm}$ & 0.49 & 1.06 & 1.00 \\
\hline
\end{tabular}




\subsection{OLED device prepared by TPBTSi}

Table 2. Experiment Medicine.

\begin{tabular}{|c|c|c|}
\hline & Manufacturer & Purity \\
\hline NPB & Aldrich & $99 \%$ \\
\hline $\mathrm{Alq}_{3}$ & Aldrich & $99.9 \%$ \\
\hline CBP & Aldrich & $99 \%$ \\
\hline BCP & Aldrich & $98 \%$ \\
\hline $\mathrm{Mg}$ & $\begin{array}{c}\text { Sinopharm Chemical } \\
\text { Reagent Co., Ltd }\end{array}$ & $99.9 \%$ \\
\hline $\mathrm{Ag}$ & $\begin{array}{l}\text { Sinopharm Chemical } \\
\text { Reagent Co., Ltd }\end{array}$ & $99.5 \%$ \\
\hline ITO Glass & $\begin{array}{c}\text { Shenzhen Nanpo.,Co., } \\
\text { Ltd }\end{array}$ & $11 \Omega /$ filtered $80 \%$ \\
\hline Acetone & $\begin{array}{c}\text { Chengdu Kelong } \\
\text { Chemical Reagent Co., } \\
\text { Ltd }\end{array}$ & $99.9 \%$ \\
\hline Ethanol & $\begin{array}{c}\text { Chengdu Kelong } \\
\text { Chemical Reagent Co., } \\
\text { Ltd }\end{array}$ & $99.9 \%$ \\
\hline
\end{tabular}

Organic multifunctional film-forming equipment OLED-V was sourced from Shenyang Vacuum Technology Institute, vertical manifold of clean bench was sourced from Nantong Cleaning Equipment Co., Ltd, spin coater KW-4A was sourced from Shanghai Chemat Function Ceramics Technology Co., Ltd. Sartorious BS electronic balance was purchased from Beijing Sartorious Mechatronics T\&H Co., Ltd, luminance meter ST-86LA and spectrometer OPT-2000 was purchased from Beijing Normal University, semiconductor test system Keithley 4200 was sourced from Keithely Company and ultrasonic cleaner KQ-200DB was bought form Nantong Ultrasonic Cleaning Equipment Co., Ltd.

Place the cleaned substrate into preprocessing vacuum chamber of the film-forming equipment Sonicel plus200 (Sonic, Korea). Prepare the oxygen plasma treatment, cleaning the surface dirt furthermore, improving surface oxygen content of ITO to increase function. Then, with no system vacuum damaging, make the vapor plating of organic materials and the electrode continuously and successively. The vacuums of vapor plating organic materials and mental electrodes is $2.1 \times 10^{-4} \mathrm{~Pa}$ and $3.8 \times 10^{-3} \mathrm{~Pa}$, the speed is $0.3-1 \mathrm{~nm} /$ and $1.1-1.3 \mathrm{~nm} / \mathrm{s}$ in order. Figure 4 shows the material's formula. Device structure is as follows: ITO/NPB $(20 \mathrm{~nm}) / \mathrm{TPBTSi}(30 \mathrm{~nm}) / \mathrm{Alq}_{3}(\mathrm{x}) / \mathrm{Mg}: \operatorname{Ag}(200 \mathrm{~nm})$, (Device A: $\mathrm{x}=0 \mathrm{~nm}$, Device B: $\mathrm{x}=10 \mathrm{~nm}$ ).

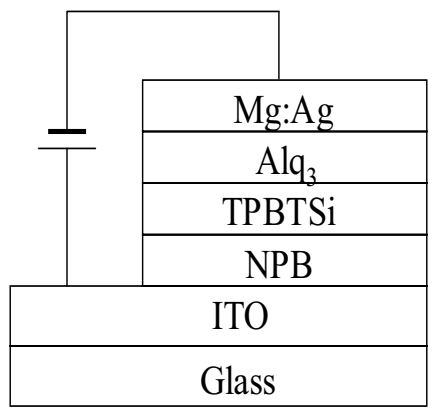

a. Device Structure Diagram

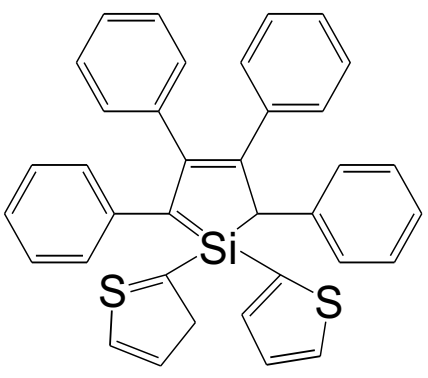

b. TPBTSi

Figure 4. Adopted Device Structure(a)and Materials Molecular Structural Formula(b). 


\section{Results and discussions}

\subsection{TPBTSi Green OLED device's performance test and analysis}

The relationship between current density and Bias voltage and the relationship between luminance and Bias voltage about double-layer (Device A) and tree-layer (Device B) TPBTSi Devices are represented in Figure 5 and Figure 6:

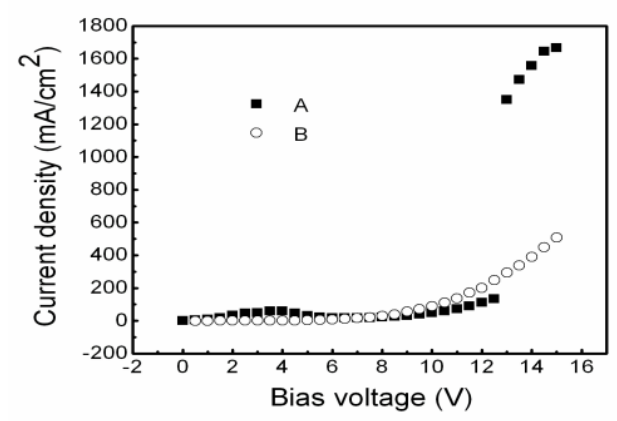

Figure 5. TPBTSi Device's $J-V$ characteristic curve.

From Figure 5 and Figure 6 , it is evident that with the same voltage, when the luminance of double-layer device is low, the maximum luminance is only $262 \mathrm{~cd} / \mathrm{m}^{2}$, at the same time the current density is only134 $\mathrm{mA} / \mathrm{cm}^{2}$. But the luminance of tree-layer device is higher than double-layer's/. Under the voltage of $15 \mathrm{~V}$, the maximum luminance reaches $7032 \mathrm{~cd} / \mathrm{m}^{2}$ with a current density of 50.7 $\mathrm{mA} / \mathrm{cm}^{2}$.

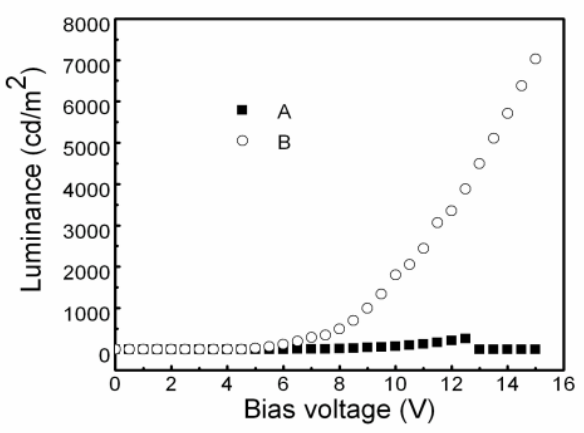

Figure 6. TPBTSi Device's $L-V$ characteristic curve.

From the characteristic curve of current density and voltage, it is evident that with the voltage of $12 \mathrm{~V}$, the current of Device A increases sharply which is caused by the number imbalance of hole and electronic current-carrying. The compound probability of carriers decreases leading to ample thermal current due to the fact that the number of the hole is greater than the electronic. With the decrease in distance between recombination zone and metal electrode /organic layer, the related exciton quenching process increases resulting in the probability of emitting photons, especially when the exciton make the radiative transition. Therefore, the transmitting capability of TPBTSi carriers is not good. In order to improve balance ability of carriers, a good film-forming electron transport material must be introduced. In addition, the current density change of Device B in which added electron transport material Alq3 is slow indicates that the effect is obvious. The electronic transports well from cathode to emitting layer through Alq3 layer and the barrier of hole from anode trough NPB/TPBTSi 
enters emitting layer too. This leads to a balance of carrier numbers, an obvious improvement of device efficiency and the device's luminous efficiency of $0.43 \mathrm{~lm} / \mathrm{W}$ when the luminance reaches $300 \mathrm{~cd} / \mathrm{m}^{2}$.

Figure 7 shows the EL spectra test result of device. The EL spectral peaks of two devices are located in the same wavelength where both are $516 \mathrm{~nm}$. Compared to NPB/TPBTSi fluorescent spectra $(\mathrm{PL})$, it is verified that this is TPBTSi characteristic spectra. From the structures of two devices, the recombination zone of hole and electronic is located in the same emitting layer and such a phenomenon is explained by energy level structure diagram of Figure 8.

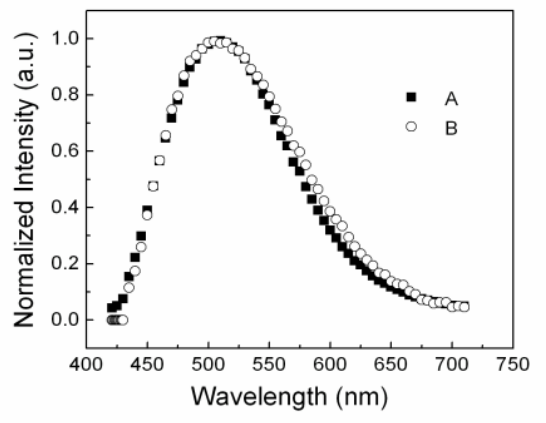

Figure 7. Device's EL spectrogram.

In the double-layer device structure, NPB plays a role of hole transport layer and the TPBTSi are both emitting layer and electron transport layer. The luminescence of device is green which is the characteristic spectrum of TPBTSi, it indicates that carrier recombination zone is completely located in TPBTSi layer. The electronic implanted into emitting layer form cathode, parts of hole implanted from anode is injected into emitting layer by overcoming the high hole barrier $(10 \mathrm{eV})$ of interface and is injected into emitting layer by Coulomb electrostatic force of space electronic charge, which forms luminescent exciton with accumulated electron from emitting layer and then sends out TPBTSi characteristic spectrum by radiative decay.
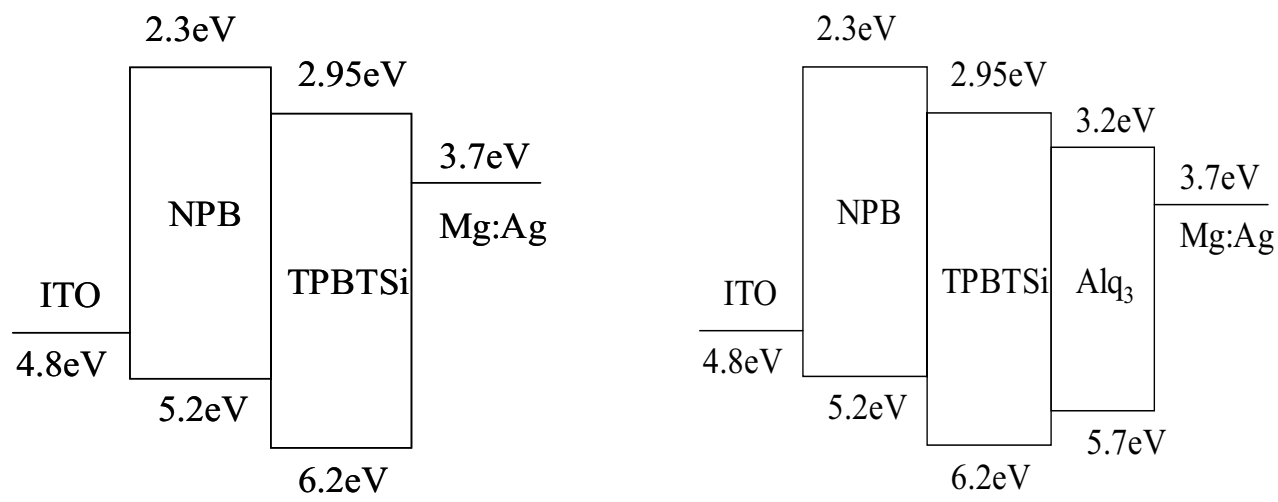

Figure 8. Device's energy level structure diagram.

In the three-layer device, the device is still with TPBTSi characteristic spectrum of green luminescence, which indicates that the compound position of carrier is in the same organic layer too. The reason for such a phenomenon is due to the fact that on the one hand, most holes can't get into $\mathrm{Alq}_{3}$ layer being blocked by highly ionization potential of TOBTISi. The $\mathrm{Alq}_{3}$ only plays a part of transporting but unsuccessful in participating in luminescing. On the other hand, the electron implanted from cathode can get into TPBTSi layer by easily penetrating into the low electronic barrier height, but with the bad electronic transmitting capacity of TPBTSi itself, the electron can't get into 
NPB layer. The NPB therefore does not participate in luminescing but only remains a part of hole transport layer. The evidence suggests that, the electron and hole are both bound in the TPBTSi layer. For the high fluorescence efficiency of this layer itself, the LUMO energy level of TPBTSi ground state molecules can take electrons easily. Furthermore the HOMO energy level loses electrons easily that results in molecules migrating into exited state and loses energy by radiative decay meanwhile gives off characteristic luminescence of TPBTSi.

\subsection{Thickness optimization of three-layer TPBTSi device}

Without the optimization of tree layer device, in order to increase the number of electronic and improve the property of device, the electronic layer $\mathrm{Alq}_{3}$ should be added into two layer device TPBTSi. To improve the luminance and efficiency, the two kinds of carriers of the device have to be kept in the best balanced state. There are two ways to keep the carriers balanced, one is to increase minority carrier, and the other is to decrease majority carrier.

(1) Increase of minority carrier

From the test of the double-layer and three-layer devices, the luminance increases fast after adding electron transport layer. But for most OLED, the hole is the major carrier. Considering the fact that the conductive capacity of NPB is relatively good, in emitting layer TPBTSi, the hole is mostly the major carrier and the electron is minor carrier. It is likely that with little number of minority carrier, there is few number of exiton which is formed by recombination resulting in low luminescence of the device. To improve the luminescence of the device, it is necessary to increase the number of electronic carrier of NPB emitting layer. On the other hand, as the conductivity of $\mathrm{Alq}_{3}$ is not as good as that of the NPB's, the change of thickness of $\mathrm{Alq}_{3}$ layer therefore influences device's electronic filed distribution and current more obviously. This in turn influences the luminescent properties of the device. For this change to be the thickness of $\mathrm{Alq}_{3}$ which is electron transport layer and preparer the light-emitting device with following structure: ITO/NPB $(20 \mathrm{~nm}) / \operatorname{TPBTSi}(30 \mathrm{~nm}) / \operatorname{Alq}(\mathrm{x} \mathrm{nm}$, $\mathrm{x}=10,20,30,40) / \mathrm{Mg}: \operatorname{Ag}(200 \mathrm{~nm})$.

The luminance-voltage $(L-V)$ diagram is showed on Figure 9. From the Figure it is evident that with the increase of the voltage the luminance of each device increases too, which presents the obvious characteristics of diode. But under the same voltage, with different thickness of $\mathrm{Alq}_{3}$ the luminance is different. When the voltage is $10 \mathrm{~V}$, the luminance of each device is as follows: (A) 1800 $\mathrm{cd} / \mathrm{m}^{2}$, (B) $1840.6 \mathrm{~cd} / \mathrm{m}^{2}$, (C) $344.6 \mathrm{~cd} / \mathrm{m}^{2}$, (D) $384.5 \mathrm{~cd} / \mathrm{m}^{2}$. Under voltage of $15 \mathrm{~V}$, the maximum luminance of each device is as follows: (A)7032.3 cd/me, (B) $9368.2 \mathrm{~cd} / \mathrm{m}^{2}$, (C) $3068.8 \mathrm{~cd} / \mathrm{m}^{2}$, (D) $3658.3 \mathrm{~cd} / \mathrm{m}^{2}$. It is obvious that when the thickness of $\mathrm{Alq}_{3}$ is larger than $20 \mathrm{~nm}$, under the same voltage, the luminance decreases obviously with the increase in thickness due to the fact that the electron injected from electrode of thinner device can easily get through recombination zone under high filed. If the $\mathrm{Alq}_{3}$ is too thick, the traps of capturing electron will get more resulting in the reduction of the compounding and colliding probabilities. And there will be a big loss of electron's capacity with a long distance transmission [10] which will reduce the number of electron reaching the recombination zone so that it will decrease the brightness (luminance) and the current. In addition, the voltages to test the device are (A)3.5 V, (B) $4.0 \mathrm{~V}$, (C)5.0 V, (D)5.5 V. From the above it is evident that the thickness of $\mathrm{Alq}_{3}$ plays a part in turn-on voltage and the voltage goes to the lowest when the thickness is $10 \mathrm{~nm}$. 


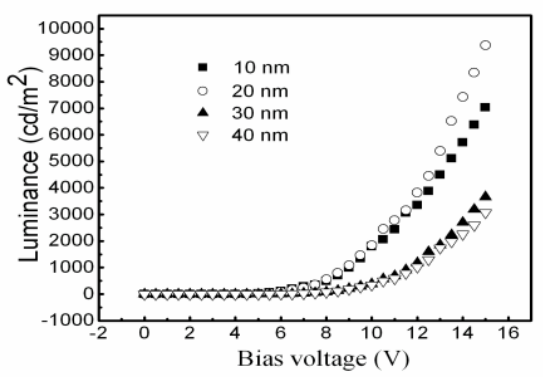

Figure 9. TPBTSi $L-V$ diagram with different $\mathrm{Alq}_{3}$ thickness.

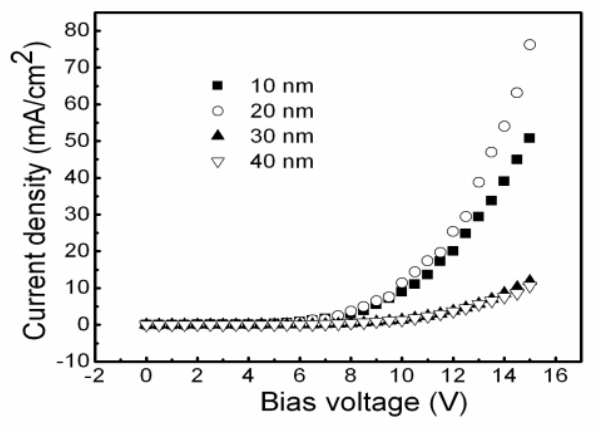

Figure 10. TPBTSi $J-V$ diagram with different $\mathrm{Alq}_{3}$ thickness.

From Figure 10, it is evident that the current density and luminance of the device has the same effect tendency even with different $\mathrm{Alq}_{3}$ thicknesses. When the $\mathrm{Alq}_{3}$ thickness is more than $20 \mathrm{~nm}$, the device current decreases obviously with the $\mathrm{Alq}_{3}$ thickness increases and when the voltage is $15 \mathrm{~V}$, the current of each device are respectively 50.7 (10 nm), 76.3 $(20 \mathrm{~nm}), 11.8(30 \mathrm{~nm}), 10.6(40 \mathrm{~nm})$ $\mathrm{mA} / \mathrm{cm}^{2}$, which has a proportional relation with the luminance (brightness) of device. It is found from the normalized EL spectrogram of device, the differences of the first three devices are little and it is hardly to observe the migrations of spectral peaks which are all kept at $516 \mathrm{~nm}$, and that is the typical TPBTSi spectral peak value. But when the thickness of $\mathrm{Alq}_{3}$ layer increased into $40 \mathrm{~nm}$, the spectral peak of the device displaced to the direction of long wave $(522 \mathrm{~nm})$ and made a redshift of $6 \mathrm{~nm}$ which means changes to the light-emitting $\mathrm{Alq}_{3}$ layer. This may be attributable to the thickness of $\mathrm{Alq}_{3}$ layer that is too big and at the same the mobility of the electron is as low as two orders of magnitude so that parts of electrons restricted in $\mathrm{Alq}_{3}$ layer lead to a recombination luminescence with the hole came from anode. Hence it is proved that the thickness of $\mathrm{Alq}_{3}$ layer used as the electron transport should be controlled within $30 \mathrm{~nm}$, and considering the current should be low (low joule while device working) and the luminescent properties should be good, the $\mathrm{Alq}_{3}$ layer is chosen of $20 \mathrm{~nm}$. 


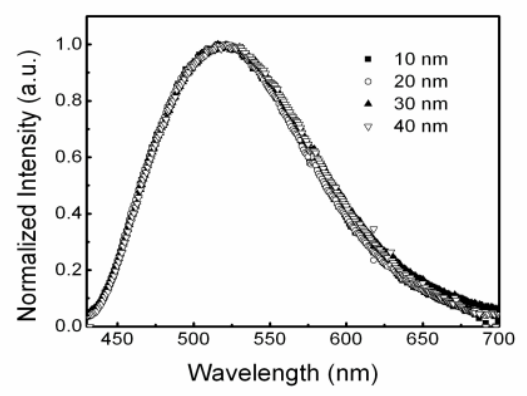

Figure 11. TPBTSi EL diagram of different $\mathrm{Alq}_{3}$ layer thicknesses.

(2) Decrease of majority carrier

After the electron transport layer is determined, to improve the property of the device by making a balance of two kinds of carriers by decreasing the number of majority carrier hole. With no thickness changes of emitting layer and $\mathrm{Alq}_{3}$ layer, prepare the devices bellowed by changing the thickness of hole transport layer: ITO/NPB(x nm, x=10,15,20,25)/TPBTSi(30 nm)/Alq $3(20 \mathrm{~nm}) / \mathrm{Mg}: \mathrm{Ag}$.

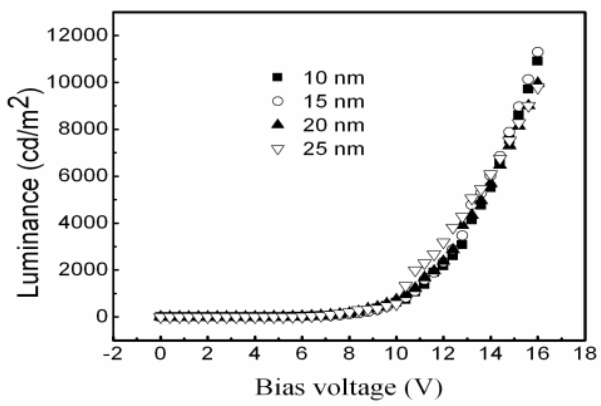

Figure 12. TPBTSi $L-V$ diagram with different NPB thicknesses.

From Figure 12 and Figure 13 it is evident that the NPB thickness change of the hole transport layer has little effect on the luminance (brightness) and current density. With the voltage of $16 \mathrm{~V}$, the luminance (brightness) and current density of the devices are respectively: $10902.8 \mathrm{~cd} / \mathrm{cm}^{2}(56.8$ $\left.\mathrm{mA} / \mathrm{cm}^{2}\right), 11290.2 \mathrm{~cd} / \mathrm{cm}^{2}\left(54.4 \mathrm{~mA} / \mathrm{cm}^{2}\right), 9960.7 \mathrm{~cd} / \mathrm{cm}^{2}\left(57.8 \mathrm{~mA} / \mathrm{cm}^{2}\right), 9780.7 \mathrm{~cd} / \mathrm{cm}^{2}(42.6$ $\left.\mathrm{mA} / \mathrm{cm}^{2}\right)$, that won't affect the efficiency and the property of the device which indicates that the hole has a high migration (shift) rate in NPB. To choose a proper thickness of the whole device, the bellowed are chosen to the best optimized structures: ITO/NPB $(20 \mathrm{~nm}) / \mathrm{TPBTSi}(30 \mathrm{~nm}) / \operatorname{Alq}_{3}(20$ $\mathrm{nm}) / \mathrm{Mg}: \operatorname{Ag}(200 \mathrm{~nm})$. 


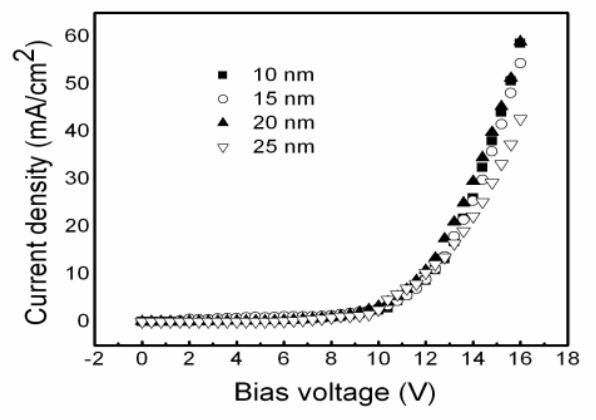

Figure 13. TPBTSi $J-V$ diagram with different TPBTSi thicknesses.

\section{Conclusions}

Using new green luminescent materials TPBTSi as emitting layer, by adding organic material $\mathrm{Alq}_{3}$ as a transport layer, to make a three layer device with structure of ITO/NPB/TPBTSi/Alq $3 / \mathrm{Mg}: \mathrm{Ag}$. The device has the maximum luminance of $11290.2 \mathrm{~cd} / \mathrm{m}^{2}$, CIE coordinate of $(0.275,0.4568)$ and the maximum luminous efficiency of $0.84 \mathrm{~lm} / \mathrm{W}$. Compared to the two layer device, the TPBTSi is a kind of luminescent material with good luminescent property but lack of the performance of electron transport. The optimized green light-emitting device can be made by adding an electron transport layer and optimizing the device by changing the organic layer's thicknesses.

\section{References}

1. T. H. Kim, H. K. Lee, O. O. Park, et al. White-light-emitting diodes based on iridium complexes via efficient energy transfer from a conjugated polymer. Adv. Funct. Mater., 2006, 15: 611-617.

2. J. Wang, Y. D. Jiang, J. S. Yu, et al. Low operating voltage bright organic light-emitting diode using iridium complex doped in 4,4'-bis[N-1-napthyl-N-phenyl-amino] biphenyl. Appl. Phys. Lett., 2007, 91: 131105.

3. X. Gong, M. R. Robinson, J. C. Ostrowski, et al. High-efficiency polymer-based electrophosphorescencet devices. Adv. Mater., 2002, 14: 581-585.

4. C. H. Lee, G. W. Kang, J. W. Jeon, et al. Blue electroluminescence and dynamics of charge carrier recombination in a vacuum-deposited poly(p-phenylene) thin film. Thin solid films, 2000, 363: 306-308.

5. C. W. Tang, S. A. VanSlyke, C. H. Chen. Electroluminescence of doped organic thin films . J. Appl. Phys., 1989, 65: 3610-3616.

6. T. Mori, K. Obata, T. Mizutani, et al. Electroluminescence of organic light emitting diodes with alternately deposited dye-doped aluminium quinoline and diamine derivative. J. Appl. Phys., 1999, 32: 1198-1203.

7. Q. Li, J. S. Yu, L. Li. High bright blue organic light emitting diodes based on a novel silole derivative. SPIE, 2007, Chengdu, 2-170, inpress.

8. A. K. Kulkarni, K. H. Schulz, T. S. Lim, et al. Dependence of the sheet resistance of indium-tinoxide thin films on grain size and grain orientation determined from X-ray diffraction techniques. Thin Solid Films, 1999, 345: 273-277.

9. H. Antoniadis, M. A. Abkowitz, B. R. Hsieh. Carrier deep-trapping mobility-lifetime products in poly(p-phenylene vinylene). Appl. Phys. Lett., 1994, 65: 2030-2032.

10. J. H. Burroughes, D. C. Bradley, A.R. Brown, et al. Light-emitting diodes based on conjugated polymers. Nature, 1990, 347: 539-541. 